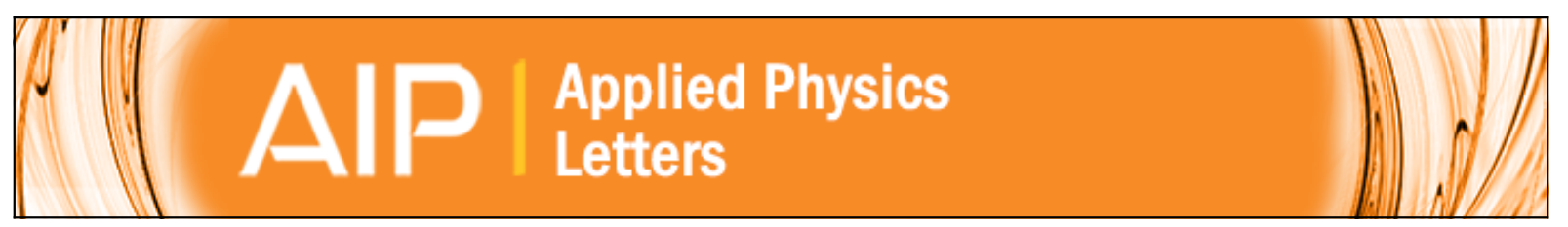

\title{
Source localization of brain activity using helium-free interferometer
}

Jürgen Dammers, Harald Chocholacs, Eberhard Eich, Frank Boers, Michael Faley, Rafal E. Dunin-Borkowski, and N. Jon Shah

Citation: Applied Physics Letters 104, 213705 (2014); doi: 10.1063/1.4880097

View online: http://dx.doi.org/10.1063/1.4880097

View Table of Contents: http://scitation.aip.org/content/aip/journal/apl/104/21 ?ver=pdfcov

Published by the AIP Publishing

\section{Articles you may be interested in}

Brain source localization: A new method based on MUltiple Slgnal Classification algorithm and spatial sparsity of the field signal for electroencephalogram measurements

Rev. Sci. Instrum. 84, 085117 (2013); 10.1063/1.4818966

High-T c superconducting quantum interference device recordings of spontaneous brain activity: Towards high-T c magnetoencephalography

Appl. Phys. Lett. 100, 132601 (2012); 10.1063/1.3698152

Data Processing Approach for Localizing Biomagnetic Sources in the Brain

AIP Conf. Proc. 922, 701 (2007); 10.1063/1.2759772

Fluctuations in Neuronal Activity: Clues to Brain Function

AIP Conf. Proc. 780, 595 (2005); 10.1063/1.2036823

Similarity regime in the brain activity

AIP Conf. Proc. 411, 299 (1997); 10.1063/1.54195

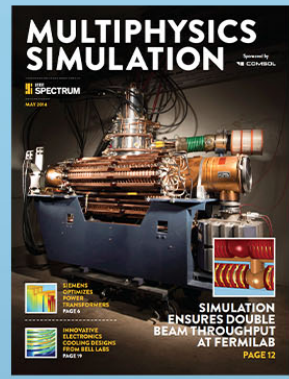

Free online magazine

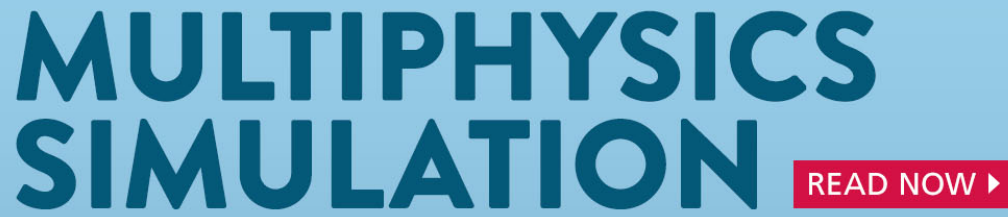




\title{
Source localization of brain activity using helium-free interferometer
}

\author{
Jürgen Dammers, ${ }^{1, \text { a) }}$ Harald Chocholacs, ${ }^{1}$ Eberhard Eich, ${ }^{1}$ Frank Boers, ${ }^{1}$ Michael Faley, ${ }^{2}$ \\ Rafal E. Dunin-Borkowski, ${ }^{2}$ and N. Jon Shah ${ }^{1,3,4}$ \\ ${ }^{1}$ Institute of Neuroscience and Medicine (INM-4), Forschungszentrum Jülich, Jülich, Germany \\ ${ }^{2}$ Peter Grünberg Institute (PGI-5), Forschungszentrum Jülich, Jülich, Germany \\ ${ }^{3}$ Department of Neurology, RWTH Aachen University, Aachen, Germany \\ ${ }^{4}$ Jülich Aachen Research Alliance (JARA)—Translational Brain Medicine, Jülich, Germany
}

(Received 21 March 2014; accepted 12 May 2014; published online 30 May 2014)

\begin{abstract}
To detect extremely small magnetic fields generated by the human brain, currently all commercial magnetoencephalography (MEG) systems are equipped with low-temperature (low- $\mathrm{T}_{\mathrm{c}}$ ) superconducting quantum interference device (SQUID) sensors that use liquid helium for cooling. The limited and increasingly expensive supply of helium, which has seen dramatic price increases recently, has become a real problem for such systems and the situation shows no signs of abating. MEG research in the long run is now endangered. In this study, we report a MEG source localization utilizing a single, highly sensitive SQUID cooled with liquid nitrogen only. Our findings confirm that localization of neuromagnetic activity is indeed possible using high- $\mathrm{T}_{\mathrm{c}}$ SQUIDs. We believe that our findings secure the future of this exquisitely sensitive technique and have major implications for brain research and the developments of cost-effective multi-channel, high- $\mathrm{T}_{\mathrm{c}}$ SQUID-based MEG systems. C 2014 AIP Publishing LLC. [http://dx.doi.org/10.1063/1.4880097]
\end{abstract}

Since the first neuromagnetic recordings, reported by David Cohen ${ }^{1,2}$ in the early seventies, magnetoencephalography (MEG) has successfully established itself as the method of choice for time-critical investigations of the spatiotemporal propagation of neural activity in neurological and psychiatric research. All current commercial MEG systems are based on low- $\mathrm{T}_{\mathrm{c}}$ superconducting quantum interference devices (SQUIDs), which require liquid helium for operation at $4.2 \mathrm{~K}$. However, the increasing number of MEG systems worldwide, and the rapidly growing number of magnetic resonance imaging (MRI) scanners in recent years, have led to a scarcity of liquid helium and consequently a dramatic price increase (about 400\%) -with further increments expected. ${ }^{3,4}$

Recently, Sander and colleagues ${ }^{5}$ have shown that optical spectroscopy-based chip-scale atomic magnetometers (CSAMs) with a magnetic sensitivity of about $200 \mathrm{fT} / \sqrt{\mathrm{Hz}}$ at $10 \mathrm{~Hz}$ are capable of recording spontaneous and somatosensory evoked neuromagnetic fields. Moreover, Johnson et al. have used this technology to measure brain responses from auditory and median nerve stimulation. ${ }^{6}$

The magnetic field resolution of commercial MEG systems based on low- $\mathrm{T}_{\mathrm{c}}$ SQUIDs is usually better than $5 \mathrm{fT} / \sqrt{\mathrm{Hz}}$ and is determined by the intrinsic SQUID noise, the Nyquist noise of the cryostat, and the magnetically shielded room (MSR). ${ }^{7,8}$ While for magnetocardiography (MCG), high- $\mathrm{T}_{\mathrm{c}}$ SQUID studies have been proven to provide sufficient sensitivity for MCG data analysis, ${ }^{9-12}$ earlier attempts to study brain signals using high- $\mathrm{T}_{\mathrm{c}}$ SQUIDs showed that the sensitivity, in particular, in the low frequency range $(<10 \mathrm{~Hz})$ of such sensors was still insufficient for source analysis of the tiny MEG signals when compared to standard low-T $\mathrm{T}_{\mathrm{c}}$ SQUIDs. ${ }^{13,14}$ Recently, Öisjöen and colleagues ${ }^{15}$ have demonstrated recordings of spontaneous human brain activity using a single- and two-channel

a)Electronic mail: J.Dammers@fz-juelich.de high- $\mathrm{T}_{\mathrm{c}}$ SQUID system with sensors placed in individual cryostats separated by more than $10 \mathrm{~cm}$, and a head-to-sensor distance of about $3 \mathrm{~mm}$. They showed field changes and spectrograms of alpha rhythms from unaveraged high- $T_{c}$ SQUID recordings. The white noise level of the high- $\mathrm{T}_{\mathrm{c}}$ SQUID employed was reported to be from approximately $50 \mathrm{fT} / \sqrt{\mathrm{Hz}}$ down to a frequency of about $10 \mathrm{~Hz}$. This level of sensitivity is not sufficient for use in whole-head systems since the typical distance between the (warm) wall of the cryostat and the subject's head usually exceeds $10 \mathrm{~mm}$ in helmet-shaped cryostats. More importantly, source analysis of neuromagnetic data using high- $\mathrm{T}_{\mathrm{c}}$ SQUIDs has not been demonstrated yet.

Very recently, Faley and colleagues ${ }^{16,17}$ showed that improvements in the fabrication of high- $T_{c}$ SQUIDs have significantly improved the sensitivity and reproducibility of high- $\mathrm{T}_{\mathrm{c}}$ SQUIDs such that liquid nitrogen-cooled SQUIDs are now comparable to low- $T_{c}$ SQUIDs in this regard. In this study, we report a neuromagnetic source localization utilizing a single-channel, high- $\mathrm{T}_{\mathrm{c}}$ SQUID. We have validated and compared our results to those obtained using a commercial low-T $T_{c}$ SQUID MEG system.

In a single subject (male, age 51, right handed), spontaneous brain activity as well as neuromagnetic field changes in response to binaural stimulation (300 trials in one run) were recorded using a single high- $\mathrm{T}_{\mathrm{c}}$ SQUID $^{16,18}$ (Fig. 1). The same experiment was repeated using the 4D-Neuroimaging Magnes 3600 WH system, a whole-head magnetometer with 248 low- $_{c}$ SQUIDs. Since we only had access to a single high- $T_{c}$ SQUID, the MEG signals in response to the auditory stimulation were recorded at 16 different locations in separate runs over the left temporal lobe. ${ }^{19}$

Resting state brain activity was measured with both open and closed eyes using both high- $\mathrm{T}_{\mathrm{c}}$ and low- $\mathrm{T}_{\mathrm{c}}$ SQUID systems. For the high- $T_{c}$ SQUID measurement, spontaneous 


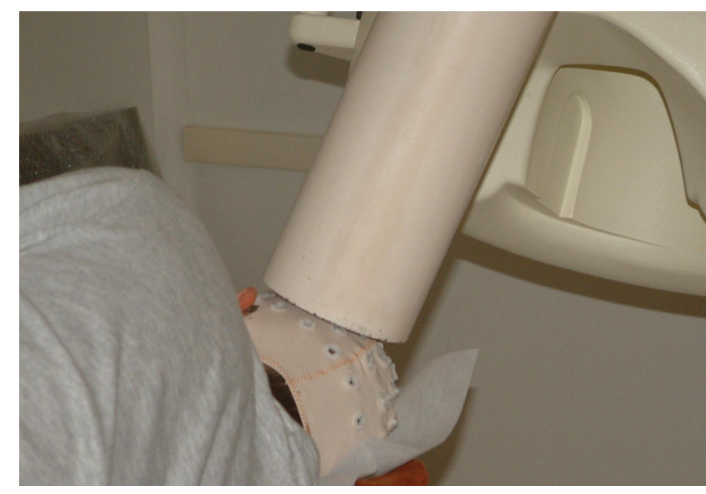

FIG. 1. Photograph showing the experimental setup during measurements of auditory evoked fields using a single high- $\mathrm{T}_{\mathrm{c}}$ SQUID. The low- $\mathrm{T}_{\mathrm{c}}$ SQUID whole head magnetometer system from 4D Neuroimaging (right side) was used for comparison.

activity was recorded at one location over the right occipital cortex, because the mounting used does not allow for central occipital recordings. Neuromagnetic fields with open and closed eyes were recorded continuously in blocks of about 9-10 s for a total duration of $3 \mathrm{~min}$. The beginning of each block, i.e., eyes open or closed, was documented by a change in the trigger code, while at the same time the subject was verbally instructed by the operator.

The time-frequency analysis (TFA) revealed clear changes in alpha power $(8-12 \mathrm{~Hz})$ for both measurements using the low- $\mathrm{T}_{\mathrm{c}}$ and high- $\mathrm{T}_{\mathrm{c}}$ SQUID systems. Figure 2 shows spectrograms for the frequency range around the alpha rhythm. The figure illustrates the de-synchronization of alpha rhythms upon opening the eyes, while during the "eyes closed" phase, the alpha power is increased dramatically, as expected. Although the experimental conditions for the high- $T_{c}$ SQUID measurement were more exhausting for the subject (the positioning of the subject was not as comfortable as compared to commercial low- $\mathrm{T}_{\mathrm{c}}$ SQUID systems), the spectrograms resemble and show a clear difference between eyes open and closed. ${ }^{20}$

Auditory evoked field responses were recorded using both the low- $\mathrm{T}_{\mathrm{c}}$ and high- $\mathrm{T}_{\mathrm{c}}$ SQUID systems, where single click tones $(50 \mathrm{~ms}$ duration, $1 \mathrm{kHz})$ were randomly presented binaurally $(\mathrm{SOA}=$ stimulus onset asynchrony $=1.8 \mathrm{~s} \pm .3 \mathrm{~s})$,

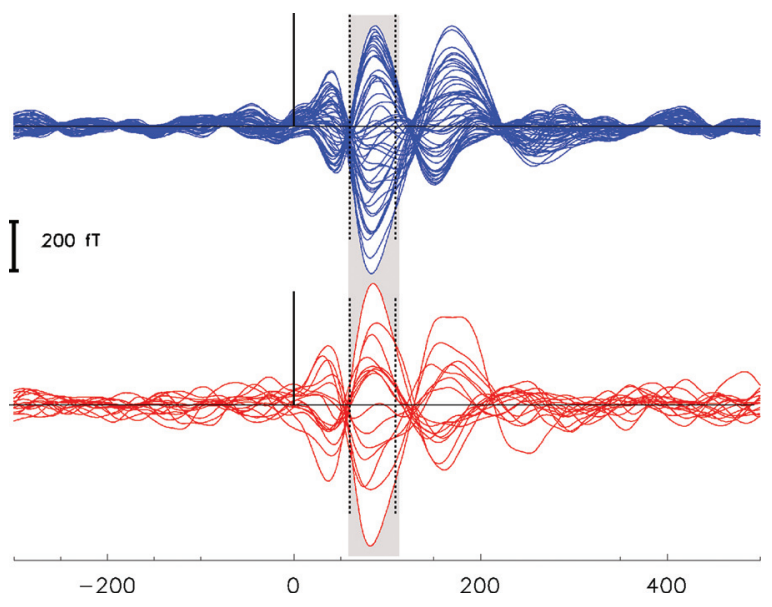

FIG. 3. MEG signal averages from the left hemisphere in response to auditory stimulation. The top row shows signal averages using 50 channels over the left temporal cortex as obtained by the whole head low- $T_{c}$ SQUID magnetometer system (blue). The bottom row shows the signals from the same subject using a single high- $\mathrm{T}_{\mathrm{c}}$ SQUID at 16 different positions (red). The time range highlighted in grey was used for source analysis.

using 300 trials for each measurement location. Neuromagnetic activity was recorded continuously with a sampling rate of $678.17 \mathrm{~Hz}$ and a bandwidth ranging from DC to $200 \mathrm{~Hz}$. All tone onsets were corrected by $-19 \mathrm{~ms}$, due to the delay of the sound delivery system, while an additional delay of $6 \mathrm{~ms}$ was subtracted for the high- $\mathrm{T}_{\mathrm{c}}$ SQUID measurement due to a constant delay of the high- $\mathrm{T}_{\mathrm{c}}$ SQUID readout electronics.

For the high- $\mathrm{T}_{\mathrm{c}}$ runs, the distance between the subject's head and the bottom of the Dewar was about 5-15 mm, while the distance between the pickup-coil and the outer Dewar wall is about $10 \mathrm{~mm}$. Hence, the total distance was approximately $20 \mathrm{~mm}$. For the low- $\mathrm{T}_{\mathrm{c}}$ measurement, the distance between the subject's head and the pickup-coils is about $23 \mathrm{~mm}$.

In both measurements, we obtained more than 290 artifact-free trials recorded over the left temporoparietal area and the entire brain region using the single channel high- $\mathrm{T}_{\mathrm{c}}$ SQUID and whole head magnetometer system, respectively. ${ }^{21}$ Figure 3 shows the MEG signal averages in response to binaural auditory stimulation. The figure shows
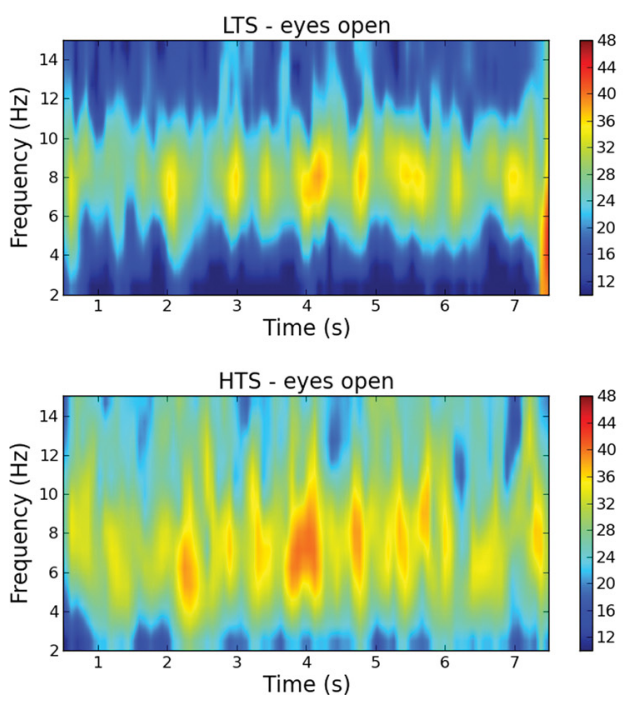
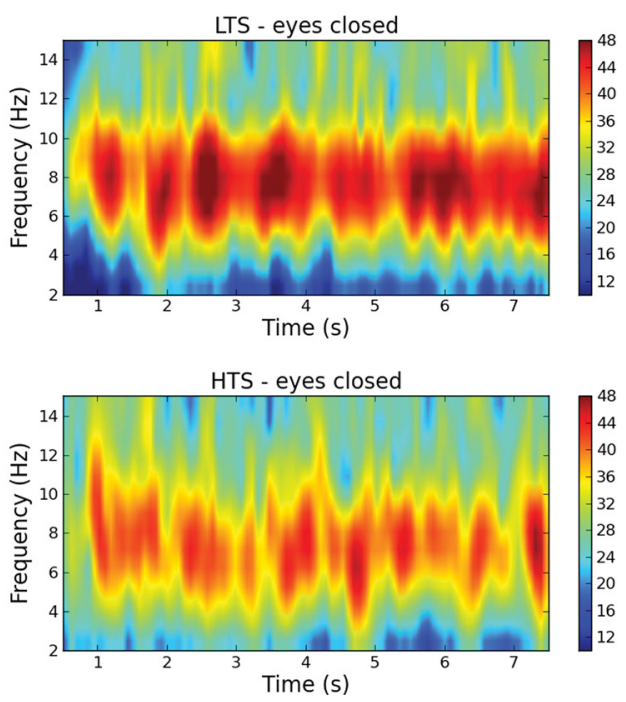

FIG. 2. Time-frequency analysis of two MEG signals recorded above the right occipital area using the low- $\mathrm{T}_{\mathrm{c}}$ (LTS) and high- $\mathrm{T}_{\mathrm{c}}$ (HTS) SQUID systems in one subject with eyes opened and closed. 


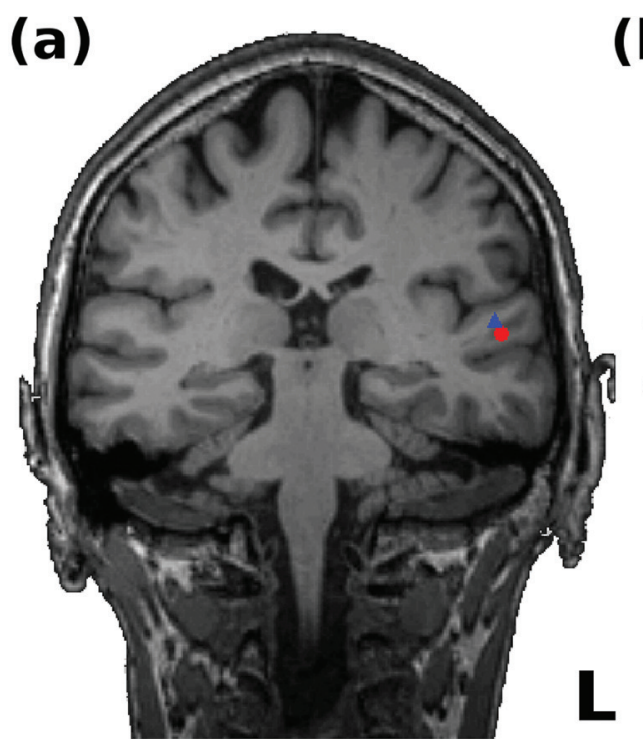

(b)

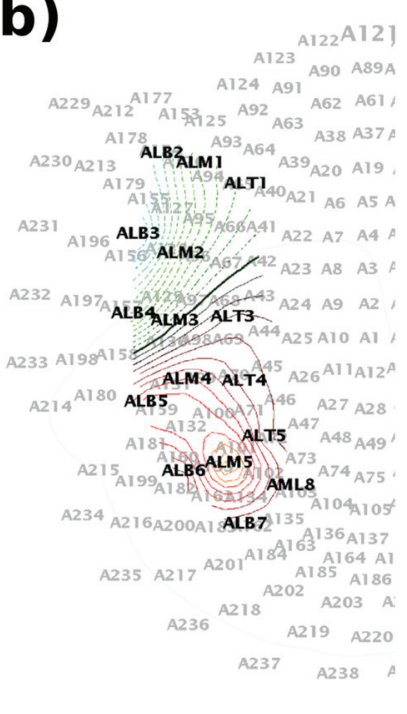

(c)

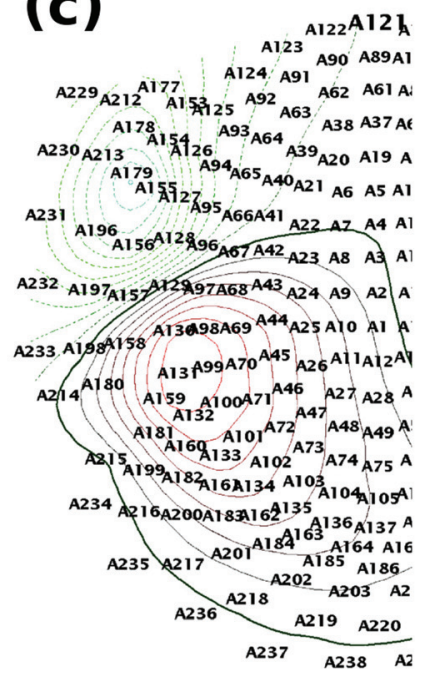

FIG. 4. (a) Source localization of auditory evoked neuromagnetic responses as recorded by high- $\mathrm{T}_{\mathrm{c}}$ (red) and low- $\mathrm{T}_{\mathrm{c}}$ (blue) SQUID systems. The location of the dipolar source from the high- $\mathrm{T}_{\mathrm{c}}$ SQUID system (red) is, as expected, in the region of the left primary auditory cortex and is in very good agreement with the source analysis using data recorded by the low-T $\mathrm{T}_{\mathrm{c}}$ SQUID system (blue). In (b) and (c), topographic field maps are shown at the time of the peak latency from averaged auditory-evoked MEG signals as recorded by the two systems, respectively. The 16 channel locations used for the high-Tc SQUID system (b) are highlighted with black bold channel labels.

clear evoked responses were recorded around $100 \mathrm{~ms}$ after stimulus onset $(0 \mathrm{~ms})$ using the high- $\mathrm{T}_{\mathrm{c}}(\mathrm{red})$ and low- $\mathrm{T}_{\mathrm{c}}$ (blue) SQUID systems. Time courses of the early (around $50 \mathrm{~ms}$ ) and late responses (around $200 \mathrm{~ms}$ ), as well as the pre-stimulus baseline, are in good agreement with the whole head low- $T_{c}$ SQUID signals.

For source localization, all trials were averaged based on stimulus onset. A single dipole fit analysis ${ }^{22}$ was performed using 50 channels and 16 positions located above the left hemisphere for the low- $T_{c}$ and high- $T_{c}$ SQUID measurement, respectively. The choice of channels for the whole head system was motivated by the pre-defined channel group "Sys:Left" from 4D-Neuroimaging, which includes 50 channels covering a large part of the temporal lobe including the area above the left auditory cortex.

Dipolar field patterns can be seen on both low- $T_{c}$ and high- $\mathrm{T}_{\mathrm{c}}$ SQUID topographic field maps (Fig. 4). The iso-contour of the evoked fields is shown for the peak latency around $100 \mathrm{~ms}$ after stimulus onset (Fig. 3). The field distribution measured by the high- $\mathrm{T}_{\mathrm{c}}$ SQUID system at 16 different positions resembles the field map of the low- $T_{c}$ SQUID system in this area and clearly covers the zero-field-line indicating the location of the source.

To localize the origin of these dipolar field maps, we applied the single dipole fit algorithm, as provided by the 4D-Neuroimaging MEG system. ${ }^{22}$ Since the low- $\mathrm{T}_{\mathrm{c}}$ SQUID measurement serves as a reference, we kept the larger number of channels for source analysis of these data.

The dipolar source was estimated using a time window of $50 \mathrm{~ms}$ ranging from 60 to $110 \mathrm{~ms}$. The dipole with the best goodness-of-fit $(\mathrm{GoF})^{22,23}$ value from the low- $\mathrm{T}_{\mathrm{c}}$ SQUID measurement was chosen for comparison and was found to be maximal at $104 \mathrm{~ms}$ after stimulus onset (Table I). Both measurements (high- $\mathrm{T}_{\mathrm{c}}$ and low- $\mathrm{T}_{\mathrm{c}}$ SQUID recordings) revealed a dipolar source that best explains the measured data by means of the best goodness-of-fit in the region of the left primary auditory cortex as expected (Fig. 4). The result for the single high- $\mathrm{T}_{\mathrm{c}}$ SQUID system is in very good agreement with the location obtained by the low- $T_{c}$ SQUID system. Table I shows that both location and direction of the source estimations are indeed comparable, where the high- $\mathrm{T}_{\mathrm{c}}$ SQUID source analysis revealed a displacement of only $7 \mathrm{~mm}$ when compared to the results obtained by the low- $\mathrm{T}_{\mathrm{c}}$ SQUID system.

Operation of MEG devices at liquid nitrogen temperatures $(77 \mathrm{~K})$ does have some significant economic and technical advantages, and both early and recent publications reported recordings of neuromagnetic brain activity utilizing high-T $T_{c}$ SQUID..$^{9,13-15}$ However, for a long time it seemed that the noise level of high- $T_{c}$ SQUID is just too high to localize magnetic brain activity. Very recently, significant improvements in the design and manufacturing of high- $T_{c}$ SQUIDs were reported by Faley and colleagues ${ }^{16,17}$ indicating that it would indeed be possible to use high- $\mathrm{T}_{\mathrm{c}}$ SQUIDs for MEG applications. In a first series of tests, we have tried to find out, if this sensor technology is suitable not only for the detection of evoked brain signals but also for the provision of a signal-to-noise ratio (SNR) sufficient for time frequency analysis and source localization of evoked brain

TABLE I. Source coordinates from single dipole analysis of auditory evoked responses using data recorded using a commercial low- $\mathrm{T}_{\mathrm{c}}$ SQUID system (using 50 channels) and a single high- $\mathrm{T}_{\mathrm{c}}$ SQUID recorded at 16 different positions above the left temporoparietal area. Coordinates are provided in the individual subject frame space, where $\mathrm{x}, \mathrm{y}$ and $\mathrm{z}$ are pointing to the nose, left ear, and top of the head. GoF refers to the goodness-of-fit quality measure. ${ }^{22,23}$

\begin{tabular}{lrrrrcc}
\hline \hline & Lat $/ \mathrm{ms}$ & $\mathrm{x} / \mathrm{mm}$ & $\mathrm{y} / \mathrm{mm}$ & $\mathrm{z} / \mathrm{mm}$ & $\|Q\| / \mathrm{nAm}$ & $\mathrm{GoF}$ \\
\hline Low- $_{\mathrm{c}}$ & 104 & -2.0 & 54.9 & 61.8 & 36.4 & 0.995 \\
High-T & 104 & -4.4 & 57.4 & 55.6 & 22.6 & 0.899 \\
Diff & 0 & 2.4 & -2.5 & 6.2 & 13.8 & 0.096 \\
\hline \hline
\end{tabular}


activity. Our results indeed confirm that the improved high-T $\mathrm{T}_{\mathrm{c}}$ SQUID technology is now suitable for MEG applications including time-frequency and source analysis.

Although atomic magnetometers seem to be a promising approach, the problem with the current technology is that its sensitivity, in particular, in the low frequency range, is currently not sufficient for source localization in MEG. Improvements of this technology have been recently reported, where the authors claim that the new atomic magnetometers provide a sensitivity better than $1 \mathrm{fT} / \sqrt{\mathrm{Hz}}$ and do not need to be shielded from Earth's magnetic field. ${ }^{24}$ To date, convincing evidence is still missing showing the capability of neuromagnetic source localization using atomic magnetometers. In contrast, SQUIDs can be configured as gradiometric superconducting flux transformers that intrinsically remove environmental noise during the measurement by recording the gradient of the magnetic flux. The advantage of using high- $\mathrm{T}_{\mathrm{c}}$ SQUIDs for neuromagnetic recordings is that long-standing experience in SQUID, SQUID readout electronics and Dewar technology exist. Therefore, a potential change from low- $\mathrm{T}_{\mathrm{c}}$ SQUID to high- $\mathrm{T}_{\mathrm{c}}$ SQUID for MEG is expected to be straightforward. Moreover, operating at $77 \mathrm{~K}$ allows, in principle, for a much thinner thermal insulation compared to low- $\mathrm{T}_{\mathrm{c}}$ SQUID systems. ${ }^{15}$ For such a system, the closer source-to-sensor distance will improve the signal-to-noise ratio, which in turn will have a positive effect on the source localization.

In conclusion, our results encourage and may pave the way for the development of multi-channel high- $\mathrm{T}_{\mathrm{c}}$ SQUID-based MEG systems. In the long term, we expect to replace low- $T_{c}$ SQUIDs with high- $T_{c}$ SQUIDs for many applications in future MEG systems. Such an upgrade would make future MEG systems independent from helium supplies, much cheaper to operate, and more user-friendly. More importantly, the availability of liquid nitrogen is guaranteed, in contrast to liquid helium.

${ }^{1}$ D. Cohen, Science 161, 784 (1968).

${ }^{2}$ D. Cohen, Science 175, 664 (1972).

${ }^{3}$ M. Peplow, Nature 497, 168 (2013).

${ }^{4}$ C. Witchalls, New Sci. 2773, 29 (2010), available at http://www.newscientist. com/article/mg20727735.700-nobel-prizewinner-we-are-running-out-ofhelium.html.

${ }^{5}$ T. H. Sander, J. Preusser, R. Mhaskar, J. Kitching, L. Trahms, and S. Knappe, Biomed. Opt. Express 3, 981 (2012).

${ }^{6}$ C. Johnson, P. D. D. Schwindt, and M. Weisend, Appl. Phys. Lett. 97, 243703 (2010).

${ }^{7}$ J. Nenonen, J. Montonen, and T. Katila, Rev. Sci. Instrum. 67, 2397 (1996).

${ }^{8}$ N. Kasai, K. Sasaki, S. Kiryu, and Y. Suzuki, Cryogenics 33, 175 (1993).

${ }^{9}$ K. Yokosawa, A. Tsukamoto, D. Suzuki, A. Kandori, T. Miyashita, K. Ogata, Y. Seki, and K. Tsukada, Supercond. Sci. Technol. 16, 1383 (2003).

${ }^{10}$ Y. Zhang, N. Wolters, D. Lomparski, W. Zander, M. Banzet, J. Schubert, H.-J. Krause, and P. VanLeeuwen, IEEE Trans. Appl. Supercond. 15, 631 (2005).

${ }^{11}$ S. H. Liao, S. C. Hsu, C. C. Lin, H. E. Horng, J. C. Chen, M. J. Chen, C. H. Wu, and H. C. Yang, Supercond. Sci. Technol. 16, 1426 (2003).

${ }^{12}$ X. H. Zeng, H. Soltner, D. Selbig, M. Bode, M. Bick, F. Rüders, J. Schubert, W. Zander, M. Banzet, Y. Zhang, H. Bousack, and A. I. Braginski, Meas. Sci. Technol. 9, 1600 (1998).
${ }^{13}$ Y. Zhang, Y. Tavrin, M. Mück, A. I. Braginski, C. Heiden, S. Hampson, C. Pantev, and T. Elbert, Brain Topogr. 5, 379 (1993).

${ }^{14}$ H.-J. Barthelmess, M. Halverscheid, B. Schiefenhovel, E. Heim, M. Schilling, and R. Zimmermann, IEEE Trans. Appl. Supercond. 11, 657 (2001).

${ }^{15}$ F. Öisjöen, J. F. Schneiderman, G. A. Figueras, M. L. Chukharkin, A. Kalabukhov, A. Hedström, M. Elam, and D. Winkler, Appl. Phys. Lett. 100, 132601 (2012).

${ }^{16}$ M. I. Faley, U. Poppe, R. E. Dunin-Borkowski, M. Schiek, F. Boers, H. Chocholacs, J. Dammers, E. Eich, N. J. Shah, A. B. Ermakov, V. Y. Slobodchikov, Y. V. Maslennikov, and V. P. Koshelets, IEEE Trans. Appl. Supercond. 23, 1600705 (2013).

${ }^{17}$ M. Faley, U. Poppe, R. D. Borkowski, M. Schiek, F. Boers, H. Chocholacs, J. Dammers, E. Eich, N. Shah, A. Ermakov, V. Slobodchikov, Y. Maslennikov, and V. Koshelets, Physics Procedia 36, 66 (2012).

${ }^{18}$ In order to calibrate the high- $\mathrm{T}_{\mathrm{c}}$ SQUID signal output, we performed noise measurements of about 2 min duration using both low- and high- $\mathrm{T}_{\mathrm{c}}$ SQUID systems, simultaneously. After downsampling to $60 \mathrm{~Hz}$, correlation analysis was applied to signal recordings from the high- and low- $\mathrm{T}_{\mathrm{c}}$ SQUID system. The low- $\mathrm{T}_{\mathrm{c}}$ SQUID sensor (A5 at the top of the helmet), which was orientated similarly to the high- $\mathrm{T}_{\mathrm{c}}$ SQUID sensor, revealed a correlation coefficient of about 0.99 . From these measurements, the signal output of the high- $\mathrm{T}_{\mathrm{c}}$ SQUID system translates to $\approx 1 \mathrm{pT} / \mathrm{mV}$.

${ }^{19}$ Each high- $\mathrm{T}_{\mathrm{c}}$ SQUID measurement location was defined by the location of the electrode position of the electroencephalography (EEG) cap from EASYCAP (EASYCAP GmbH, Berlin, Germany), which the subject was wearing without EEG electrodes. Before the measurement, the subject's head, including the electrode locations of the EEG cap, was digitized using a 3D digitizer (Polhemus, 3Space/Fastrak, USA) to define a head coordinate system. For a comparison of the source analysis between the two systems, coordinates from the high- $\mathrm{T}_{\mathrm{c}}$ SQUID measurements were aligned with one of the low- $T_{c}$ SQUID sensors. This was done by minimizing the distance between a line normal to the low- $\mathrm{T}_{\mathrm{c}}$ SQUID sensor and the electrode location from the EEG cap where the high- $\mathrm{T}_{\mathrm{c}}$ SQUID sensor was placed. To correct for the high- $T_{c}$ SQUID sensor-to-head distance, each sensor coordinate was shifted in a normal direction by $20 \mathrm{~mm}$ (which was the approximate head to high- $\mathrm{T}_{\mathrm{c}}$ SQUID sensor distance), assuming the electrode locations are distributed on a spherical surface. With this procedure, we derived a virtual sensor configuration comprising all high- $\mathrm{T}_{\mathrm{c}}$ SQUID channel positions.

${ }^{20}$ TFA was applied using the Python derivate of the MNE toolbox (http://martinos.org/mne). Each period of the task "eyes open" and "eyes closed" was limited to $7 \mathrm{~s}$; to avoid interference due to the instruction of the task change, $1 \mathrm{~s}$ at the beginning and one at the end of each block was skipped. For each condition (eyes open vs. eyes closed), a total of 9 epochs with $7 \mathrm{~s}$ duration were used to calculate an average spectrogram. For comparisons between the two systems, the MEG signals were normalized to unit variance prior to analysis.

${ }^{21}$ Artifacts in the signal of the high- $\mathrm{T}_{\mathrm{c}}$ and low- $\mathrm{T}_{\mathrm{c}}$ SQUID recordings were identified by using a peak-to-peak measure applied to the full data set using a sliding window of $200 \mathrm{~ms}$ length. With respect to the signals recorded with the whole head system, we used a peak-to-peak threshold of $\geq 8 \mathrm{pT}$, whereas a threshold of about $15 \mathrm{pT}(\triangleq 15 \mathrm{mV})$ was used for the high- $T_{c}$ SQUID recordings. The reason for the lower threshold used for the low- $T_{c}$ SQUID system is that data recorded by that system include an online noise compensation. After artifact removal, MEG data from the auditory experiment were bandpass filtered from 3 to $30 \mathrm{~Hz}$ including a notch filter at the power line frequency of $50 \mathrm{~Hz}$. Trials around stimulus onset were defined with $1 \mathrm{~s}$ length and a pre-stimulus time of $400 \mathrm{~ms}$. On average, 293 and 294 trials were left after discarding trials with artifacts or unusually large amplitudes for the low- and high- $\mathrm{T}_{\mathrm{c}}$ SQUID recordings, respectively.

${ }^{22}$ T. Lypchuk, Dipole Fit Algorithms for MEG Analysis (Biomagnetic Technologies, Inc., San Diego, USA, 1990).

${ }^{23} \mathrm{GoF}=1-\left\langle\left(m_{\text {meas }}-m_{\text {fit }}\right)^{2}\right\rangle /\left\langle\left(m_{\text {meas }}\right)^{2}\right\rangle$.

${ }^{24}$ D. Sheng, S. Li, N. Dural, and M. V. Romalis, Phys. Rev. Lett. 110, 160802 (2013). 